\title{
Interdisciplina, cultura visual y educación artística en Chile: de la prueba estandarizada a la invisibilización de las artes en la escuela
}

\author{
Interdiscipline, visual culture and artistic education in Chile: from the \\ standardized test to the invisibilization of the arts in school \\ Interdisciplina, cultura visual e educação artística no Chile: do teste \\ padronizado à invisibilização das artes na escola
}

\begin{abstract}
Patricia Fernanda Quintana Figueroa
Candidata a Magíster en Educación Artística Docente Programa Formación Pedagógica Universidad Austral de Chile Profesora de Artes Visuales, Liceo Industrial de Valdivia/Instituto Italia de Valdivia, Chile patricia.quintana@uach.cl
\end{abstract}

\section{Resumen}

El siguiente artículo, surge a partir de un proyecto con enfoque interdisciplinar realizado en un Liceo Industrial público de la ciudad de Valdivia, Chile. El Proyecto en un inicio diagnostica la fuerte desvaloración de la asignatura de artes visuales dentro del contexto de la educación técnica, incluyendo la idea de asignatura complementaria o de segunda categoría, ya que no está dentro de ningún tipo de evaluación estandarizada. Se realiza una mirada acerca de la visión que tiene el Ministerio de Educación en relación al enfoque interdisciplinar y la importancia de éste en la escuela. Se consideran los nuevos cambios en las bases curriculares de artes visuales a partir del año 2014, donde la interdisciplina y la cultura visual son perspectivas claves en el desarrollo de los programas de futuros estudios para la enseñanza secundaria. A partir de las reflexiones, se ejemplifica el desarrollo de la interdisciplina y la comprensión de la cultura visual a través de un proyecto de arte y física con un enfoque interdisciplinar, con el objetivo de cambiar la percepción de estas disciplinas en los y las estudiantes, a través de un taller que abordará los temas relativos a la luz y su uso en la fotografía y la serigrafía.

Palabras claves: Educación artística, currículo, disciplina, artes visuales, interdisciplina, cultura visual. 


\begin{abstract}
The following article arises from a project with an interdisciplinary approach in a Public Industrial Lyceum of the city of Valdivia, Chile. The Project initially diagnoses the strong devaluation of the Visual Arts Subject within a context of Technical Education, including the idea of complementary subject or second category since it is not within any standardized evaluation. It looks at the vision of the Ministry of Education in relation to the interdisciplinary approach and the importance of this in the school. The new changes in the curricula of visual arts are considered as from 2014, where interdisciplinary and visual culture are key perspectives in the development of programs of future studies for secondary education. From the reflections, we exemplify the development of interdiscipline and the understanding of visual culture through an art and physics project with an interdisciplinary approach, aiming to change the perception of these disciplines in students, through a workshop that will address the issues related to light and its use in photography and screenprinting.
\end{abstract}

Keywords: Arts education, curriculum, discipline, visual arts, interdisciplinary, visual culture.

\title{
Resumo
}

O seguinte artigo surge de um projeto com abordagem interdisciplinar em um Liceu Industrial Público da cidade de Valdivia, no Chile. O Projeto inicialmente diagnostica a forte desvalorização do Assunto das Artes Visuais em um contexto de Educação Técnica, incluindo a idéia de assunto complementar ou segunda categoria, uma vez que não está em uma avaliação padronizada. Ele analisa a visão do Ministério da Educação em relação à abordagem interdisciplinar e a importância disso na escola. As novas mudanças nos currículos das artes visuais são consideradas a partir de 2014, onde a cultura interdisciplinar e visual são perspectivas-chave no desenvolvimento de programas de estudos futuros para o ensino secundário. A partir das reflexões, exemplificamos o desenvolvimento da interdisciplina e a compreensão da cultura visual através de um projeto de arte e física com abordagem interdisciplinar, com o objetivo de mudar a percepção dessas disciplinas em estudantes, Através de uma oficina que abordará as questões relacionadas à luz e seu uso na fotografia e na serigrafia.

Palavras-chave: Educação artística, currículo, disciplina, artes visuais, interdisciplinar, cultura visual.

\section{Introducción}

Para concebir una educación de calidad en Chile se utiliza una serie de instrumentos que miden procesos educativos tanto para estudiantes como profesores. A los estudiantes se les mide en las escuelas con pruebas estandarizadas o adecuadas al contexto, que se dirigen básicamente a los aprendizajes de los contenidos pasados por profesores y profesoras según los programas curriculares vigentes. A nivel nacional tenemos los instrumentos de evaluación más importantes, por un lado, durante la enseñanza básica y secundaria, 
existe el Sistema de Medición de la Calidad de la Educación (SIMCE), que busca generar datos específicos en lenguaje, matemática, ciencias sociales y ciencias naturales, cuyos resultados son utilizados para posicionar a las escuelas en diversos escalafones según criterios de vulnerabilidad y conocimientos de estudiantes de educación básica y Media. Por otro lado, al finalizar los estudios secundarios, los estudiantes pueden presentar la prueba de selección universitaria (PSU), la cual les permite ingresar a las Universidades del Consejo de Rectores de Chile, y a su vez permite visibilizar los colegios con mejor rendimiento en relación a lenguaje, matemática, ciencias sociales y ciencias naturales (física, química y biología). Hasta hace pocos años atrás, las mediciones estaban adscritas y enfocadas a visibilizar los conocimientos adquiridos por estudiantes de todo el país. Desde el año 2003, entre estos instrumentos evaluativos se suma, la evaluación docente, la cual permite conocer el nivel de preparación de profesoras y profesores.

Si realizamos un breve recuento, la forma en que se ha implantado la medición de calidad a la educación pública y subvencionada del país, ha sido única y exclusivamente a través de diversos instrumentos, que buenos o malos, arrojan criterios y resultados estadísticos y cuantificables. Con estas formas evaluativas se busca dar cuenta de habilidades, procedimientos, contenidos y destrezas en distintos ámbitos medidos por rúbricas estandarizadas. Gracias a la relevancia que se dan a estos instrumentos en la escuela, se han invisibilizado otras áreas del conocimiento dentro del contexto escolar. Esto a su vez ha posicionado el pensamiento racional sobre la dimensión emocional, este último vinculado con la enseñanza de las artes, lo que ha producido un profundo desequilibrio entre ambas dimensiones (Magendzo en Mora y Osses, 2012) Este desequilibrio entre la dimensión emocional y racional produce que exista un reduccionismo e infantilización de la educación artística (Mora y Osses, 2012). Debemos considerar en Chile, que la educación artística no participa de ninguna prueba estandarizada (CNCA, 2016), no se estima con base en criterios estandarizados, por ello, no es cuantificable, no arroja resultados concretos ni para estudiantes ni para las escuelas, lo que conlleva a situarla al final de la pirámide jerárquica (CNCA, 2016). $\mathrm{Se}$ ha reducido entonces, como una asignatura complementaria (Errázuriz, 2001). Quedan las Artes Visuales como una disciplina subvalorada, incluso entre profesores se considera que es menos importante.

Es así como los procesos de educación artística y específicamente las artes visuales, han buscado de diferentes formas la validación dentro del curriculum escolar desde la libre expresión liderada por Key, el enfoque cognitivista postulado por Einser, el lenguaje visual y la alfabetización propuesta por Dondis, la búsqueda de equilibrio de Barbosa, hasta el enfoque de la comprensión de la cultura visual postulada por Fernando Hernández (Augustowsky, 2012, p.157-167). Todos han aportado para dar respuestas al olvido de las artes en la educación y a reposicionar esta área dentro del marco del conocimiento, sacándola del olvido en el que ha caído, víctima del exitismo y el estándar (Gaete y Miranda, 2007).

En este contexto, es que hoy, el currículo actual chileno, se encuentra en un proceso 
de cambio, existen nuevas bases curriculares para la enseñanza de las artes visuales en enseñanza básica y parte de la enseñanza secundaria, sustentándose en perspectivas relacionadas a la creatividad, la cultura, la difusión, la inclusión, la interdisciplina, la tecnología y la cultura visual (Mineduc, 2015).

\section{Curriculum disciplinario, las artes visuales en relación a otras disciplinas}

En el campo de las asignaturas y disciplinas que existen dentro del currículo escolar en Chile, estas se dividen según el área del conocimiento. El discurso oficial que promueve el Ministerio de Educación, es que esta división disciplinar surge a partir de la escuela moderna:

Con el surgimiento de la escuela moderna, la separación disciplinar se convirtió en un modo práctico de organizar el conocimiento y los contenidos que se enseñaban. Esta organización curricular en asignaturas se mantiene hasta hoy en día en las escuelas tradicionales de Chile y gran parte del mundo. Las disciplinas son áreas del conocimiento que se distinguen por su tema de estudio. Para abordarlo crean conceptos, lenguajes, metodologías y teorías especializadas que las distinguen entre sí. En el caso de las artes, estudian y producen manifestaciones artísticas y culturales como fiestas y bailes, obras teatrales y/o escénicas, obras visuales y musicales, poesía o artesanía, utilizando diversas técnicas: pictóricas, de danza, de escultura, de actuación, entre otras. Los conceptos de las artes refieren a los modos de representación, al rol del espectador frente a la obra de arte o a las dinámicas de circulación de los bienes simbólicos, los cuales permiten la construcción de metodologías y/o teorías en torno a su estudio, como la historia del arte, las teorías de interpretación de obras artísticas o la teoría de la música. Se reconocen dos grandes campos disciplinares: Ciencias, en referencia a las llamadas ciencias exactas y ciencias descriptivas. Humanidades, conjunto de estudios y disciplinas referentes a la cultura humana. Además de los distintos lenguajes artísticos engloba a las ciencias sociales, que estudian de forma sistemática procesos socioculturales como la historia y geografía, la sociología, la antropología y la psicología. (CNCA, 2016, cuaderno No. 3, p.14)

Es así, como hoy en las escuelas públicas y subvencionadas de Chile, se trabaja con base en esta división, fragmentando las disciplinas y situándolas en esferas distintas. Esto sumado a la jerarquización y la estandarización de la educación, genera que las ciencias humanas sean consideradas muy importantes pero reconociendo que éstas a veces se consideran como menos importantes que las ciencias naturales:

Pese a que en ocasiones se ha considerado a las humanidades y las ciencias sociales como saberes de menor rigor científico y, por lo tanto, menos importantes, en la actualidad se reconoce la relevancia de estos ámbitos y sus aportes para una mejor comprensión de la experiencia humana (CNCA cuaderno $\mathrm{n}^{\circ} 3,2016$, p.14).

Es entonces que finalmente todo el énfasis que se le da a la educación académica y técnica termina por relegar a las humanidades, y sobre todo a las artes a una categoría inferior dentro de los programas de estudios, lo que favorece el 
divorcio entre las artes y la ciencia. (Bamford, 2009).

Y si el arte ha logrado de alguna forma tener espacio en el ámbito educativo, es sólo por el hecho de que permite el desarrollo de técnicas y habilidades, con el fin de aprender cosas útiles (Errázuriz, 2001) en otras asignaturas a las que se les da más relevancia en los planes de estudio como son las matemáticas y el lenguaje. A pesar de todos los intentos, se ha visto que la enseñanza artística tiene un carácter funcional.

El currículo en la educación artística actualmente se destaca por ser flexible, ya no es obligatorio en la enseñanza media, y se puede elegir entre artes musicales o artes visuales en las escuelas que posean un currículo científico-humanista. (Cobos, 2010). Esta situación también aporta a que la asignatura sea menos relevante, ya que al ser optativo, la evaluación deja de importar (Errázuriz, 2002). Esta situación se agudiza aún más en los Liceos Técnicos, ya que artes visuales $\mathrm{o}$ artes musicales sólo se considera hasta segundo año de educación media, dejando los dos últimos años de secundaria, sin educación artística.

\section{Artes visuales, interdisciplina y cultura visual.}

La interdisciplina y la cultura visual son dos de las perspectivas que las nuevas bases curriculares de artes visuales están promoviendo y proponiendo como ejes de acción en la enseñanza de esta. Desde el Ministerio de Educación, el enfoque interdisciplinar se comprende como "la cooperación entre dos o más disciplinas, sin la fragmentación propia de lo disciplinar, para abordar un tema, objeto o problemática mediante sus métodos específicos, de modo que se enriquezcan mutuamente y desarrollen conocimientos más complejos y profundos" (Cuaderno $\mathrm{n}^{\circ} 3$, caja de herramientas de Educación Artística, p.17). A su vez una educación que tenga cimientos en el enfoque interdisciplinar, permitirá construir un conocimiento complejo, dinámico y localizado; abordar y considerar los entornos culturales de niños, niñas $\mathrm{y}$ jóvenes; motivar a los y las estudiantes, atendiendo sus inquietudes e intereses; desarrollar proyectos colaborativos que incluyan la participación de diferentes personas pertenecientes a una institución. (CNCA, 2016)

Otra esfera y perspectiva fundamental de las nuevas bases curriculares de las artes visuales es la comprensión de la cultura visual, en este sentido Hernández (1996) plantea que no se trata de exponer al estudiante solo al conocimiento formal, conceptual y práctico en relación a las artes, sino que considera importante tener en cuenta diferentes pueblos, manifestaciones y sociedades como parte de la cultura visual. "Este enfoque comprensivo trata de favorecer en los estudiantes una actitud reconstructiva, es decir, de autoconsciencia de su propia experiencia en relación con las obras, los artefactos, los temas o los problemas que trabajan en clase. Para ello el desarrollo de estrategias para la comprensión resulta fundamental" (Hernández, 1996, p. 18).

La cultura visual así entendida cumple con la función de manufacturar las experiencias de los seres humanos, mediante la producción de significados visuales, sonoros, estéticos que contribuyen a la construcción de la conciencia individual y social, mediante la incorporación de los indicios visuales con valor simbólico producidos por grupos diferentes (los artistas serían uno 
de ellos) en el proceso del intercambio social. (Augustowsky, 2012).

Según Hernández "la idea básica de la cultura visual implica que no existe un entorno sociocultural que tenga identidad independiente de cómo los seres humanos se valen de los significados y los medios tecnológicos y comunicacionales mediantes los cuales cada uno construye su subjetividad y su vida mental" (Augustowsky, 2012, p.165).

Es así como se pretende dentro de la institución escolar, potenciar ambas perspectivas para favorecer una educación de calidad, desenmarcando los procesos de aprendizaje y educativos de un enclave solamente medido por estándares, puesto que éstos hasta hoy sólo han promovido la competencia como base fundamental del proceso educativo.

\section{Proyecto con enfoque interdisciplinar y comprensión de la cultura visual: desde las artes visuales a la física/desde la física a las artes visuales}

En la actualidad, laboro como profesional en dos ámbitos educativos: educación superior y educación secundaria. Con relación a la educación secundaria, me desempeño en el Liceo Industrial de Valdivia, cuyo objetivo es formar técnicos de nivel medio en las áreas de mecánica, construcción y electricidad. La educación técnica se puede considerar como un subsistema del sistema educativo formal, que tiene por objetivo preparar la fuerza de trabajo calificada de nivel medio que el país requiere para el desarrollo de sus planes económicos y sociales. Es importante mencionar que en particular, quienes cursan la educación técnico profesional provienen de los quintiles inferiores de ingresos y reportan indicadores de logro educacional de entrada más bajos en relación a aquellos que siguen la Enseñanza Media Científico Humanista (Mineduc, 2012:3-4).

Es así como la educación artística, se ha convertido en un trámite obligatorio, que no reporta mayor incidencia para estudiantes, cuya formación está ligada al ámbito técnico y del trabajo y curricularmente, como ya se ha mencionado sobre los Liceos Técnicos en Chile, sólo imparten artes hasta segundo año medio, y se les obliga (en este caso en particular no tienen la opción de decidir), a estar en artes musicales o artes visuales.

Es por ello, que, como docente de artes visuales, he experimentado la situación de desmedro y desventaja en que se encuentra la asignatura, dentro de un establecimiento técnico. Entonces ¿Qué hacer? Como también mi especialidad es la fotografía, decidí conversar con la profesora de física, para ver si podíamos entablar un dialogo en torno a nuestros intereses, y por sobre todo, una discusión en relación a la búsqueda de una educación de calidad y con principios de equidad pensando que nuestros estudiantes poseen un $84 \%$ de vulnerabilidad y que hay manifestaciones y conocimientos que muy difícilmente estarán a su alcance.

Partimos de diversas reflexiones sobre nuestra propia práctica pedagógica, las observaciones, las dinámicas y relaciones que he podido observar en relación a las artes visuales y los/as estudiantes. Luego logramos encontrar un punto común: Ambas asignaturas solo duraban 2 años en la secundaria, y sólo tenían 2 horas a la semana para su aprendizaje.

Empezó entonces la búsqueda de plantear un proyecto que se sustentara en el tiempo y que a su vez, pudiera ser 
financiado. ¿y cómo llegamos a la Fotografía?

Según el estudio internacional sobre el impacto de la educación artística de Anne Bamford, en más del $90 \%$ de los países participantes de dicho estudio, consideran la pintura, la música y el dibujo dentro de las disciplinas más conocidas de la educación artística, en cambio, los nuevos medios como la fotografía y el cine están presentes en el $50 \%$ de los países aunque se observa una fuerte concentración en los países más avanzados económicamente (2009).

Solamente, el $50 \%$ de los países consideraban estos nuevos medios, tan utilizados por nuestros y nuestras estudiantes, dentro de su malla curricular. Así decidimos trabajar en conjunto teniendo como enfoque, la interdisciplina y nuestra didáctica basada, en el interés crítico cuya finalidad es la reflexión y elaboración de saberes proclives al logro de la autonomía, la emancipación y la transformación de las prácticas sociales.

(Bazán y Astorga, 2012:56). Dentro de nuestra propuesta didáctica, debimos considerar como base fundamental y guía para el desarrollo de nuevos saberes, la comprensión de la cultura visual, la cual en la era de las nuevas tecnologías, busca no sólo formar receptores de imágenes sino también productores de sentidos y significados (Augustowsky, 2012).

Finalmente como resultado de estas reflexiones, se ha implementado un laboratorio de luz, cuyos objetivos son generar una nueva visión curricular con enfoque interdisciplinar de las artes y las ciencias. El laboratorio de luz del Liceo Industrial de Valdivia, Chile; vinculó curricularmente la asignatura de artes visuales y física; y estableció como objetivos: implementar un laboratorio de luz para el desarrollo de talleres artísticos-científicos con enfoque interdisciplinar; contribuir al cambio de percepción de los estudiantes en relación a las artes visuales y contribuir al proceso de comprensión de la cultura visual a través de las diversas miradas que nos plantea la interdisciplina.

Este proceso se ha llevado a cabo durante un año, donde se ha logrado dialogar entre profesoras, estudiantes y parte de la comunidad educativa en torno a la importancia de generar estos espacios de interdisciplinariedad en las escuelas, y más aún, en los Liceos Técnicos, cuya participación en la educación artística es escasa. Se ha trabajado en un laboratorio oscuro realizado artesanalmente, y se ha vivido la experiencia de conocer los espacios, el territorio, la cultura a través de cámaras digitales y artesanales. Todo esto, ha logrado una simetría de ambas disciplinas, y un cambio en la percepción de las asignaturas de quienes participan del taller, valorando $\mathrm{y}$ visibilizando nuevamente a las artes visuales dentro de su proceso de formación.

$\mathrm{Si}$ bien, hemos logrado establecer un discurso que supera de cierta forma a las artes visuales y a la física, estableciéndolas como asignaturas complementarias entre sí, y de igual importancia y relevancia para el proceso educativo de los estudiantes con los cuales trabajamos, este se ha quedado en ese espacio y aún no es un discurso que permee a la institución por completo. Se comprende ya que como menciona Gaete y Miranda (2006) que se ha intentado formar una idea que las reformas tendrán grandes cambios, aún no se vislumbran esos cambios, todavía no hay un discurso consensuado y establecido acerca de la interdisciplina y los currículos aún se 
basan en objetivos más objetivos menos, contenidos más, contenidos menos.

\section{Conclusiones}

Hoy día es muy complejo establecer un dialogo y una discusión de la importancia de las artes en la educación, y es precisamente por eso ¿por qué debemos argumentar la importancia? ¿Por qué a las matemáticas no se les pregunta para que sirven? Quizá si debamos recuperar las artes como espacio de conocimiento recuperar la noción de arte como un saber para otorgar mayor presencia a las disciplinas artísticas en los currículos de la educación formal (Einser en Augustowsky, 2012). Potenciar la educación estética en la escuela (Errazuriz, 2006) y desempolvar todo ese conocimiento erudito, que nos podría dar una esperanza de reposicionarnos ante un discurso jerárquico que nos invisibiliza como disciplina, infantilizándonos y relegándonos como una aporte al regalo del día de la madre, a la corbata de papel para el día del padre y al viejito pascuero con cascara de huevo y algodón para la navidad.

Es posible entonces, poner en discusión, poner nuevamente a las artes en la educación, como parte de ella, al igual que todas las demás áreas del conocimiento, que conviven en un mismo espacio institucional, y están ahí con el mismo fin: aportar a la transformación social a través de la construcción de conocimiento.

Actualmente el proyecto anteriormente mencionado está llegando a su fin, y veremos si los resultados de dicho intento de trabajar en un Liceo Técnico, con un enfoque interdisciplinar, basándonos en una didáctica de interés crítico, y promoviendo de alguna $\mathrm{u}$ otra forma la comprensión de la cultura visual desde la escuela y desde las Artes, tendrán o no frutos. Esperamos que a largo plazo los tenga, y se demuestre que la praxis transformadora es aquella que se adquiere a través del compromiso y la búsqueda de nuevas formas de relacionarse en el aula.

Finalmente debemos comprender que la búsqueda de una educación de calidad debe urgentemente separarse de las pruebas estándar, las cuáles miden con la misma vara a nuestros estudiantes del Norte, del Centro, de Sur, a nuestros estudiantes cuyas realidades sociales son múltiples y diversas. "No basta con elevar algunos índices que miden el rendimiento escolar, aunque sea de acuerdo a criterios internacionales, para afirmar que estamos mejorando la calidad de la educación en Chile. Un mayor rendimiento en matemáticas y/o lenguaje no significa que hayamos alcanzado una buena educación" (Errázuriz, 2006:19).

\section{Referencias}

Augustowsky, G. (2012) El Arte en la enseñanza, Editorial Paidós.

Bamford, A (2009) El factor ¡wuau!, el papel de las Artes en la Educación, Barcelona, Editorial Octaedro.

Bazán, D. Astorga, B (2011) Introducción a una didáctica crítica, democratizadora y comprometida con la transformación social. Revista Diatriba $N^{\circ}$ 1, Editorial Quimantú.

Cobos, D. (2010). Innovación y creatividad en las Artes Visuales: las claves de la reivindicación. Extraído en septiembre 2016 de la página web: http://www.udg.edu/portals/3/didactiqu es2010/guiacdii/ACABADES\%20FIN ALS/284.pdf 
Consejo Nacional de la Cultura y las Artes (2016) Caja de herramientas para la Educación Artística, Santiago, MINEDUC.

Errázuriz, L. (2001) Como evaluar el Arte, Santiago, Ediciones PUC.

Errázuriz, L. (2006) Sensibilidad Estética, un desafío pendiente en la Educación Chilena, Ediciones PUC.

Gaete, M. Miranda, L (2006) Arte y Filosofía en la Escuela chilena: Entre el desarraigo y el olvido. Extraído agosto 2015 de la página web: http://repositorio.uchile.cl/bitstream/ha ndle/2250/122980/Desarraigo_y_Olvid o.pdf? sequence $=1$

Hernández, F. (1996) Educación Artística para la comprensión de la cultura Visual. Artículo extraído en Agosto 2016, de la página web: http://publica.webs.ull.es/upload/REV \%20QURRICULUM/12$13 \% 20 \% 201996 / 03 \% 20$ (Fernando\%2 0Hern\%C3\%A1ndez).pdf

Ministerio de Educación (2015) Bases curriculares de $7^{\circ}$ básico a $2^{\circ}$ medio Artes Visuales. Extraído en agosto 2016 de la página web: www.curriculumenlineamineduc.cl

Ministerio de Educación (2012). Educación Técnica Profesional en Chile. Extraído en septiembre 2016 de la página web: http://portales.mineduc.cl/usuarios/min educ/doc/201204241130130.DiagnOsti coEducaciOnTPCentrodeEstudiosMIN EDUC.pdf

Mora, J. Osses, S. (2012) Educación Artística para la formación integral.
Complementariedad entre Cultura Visual e identidad juvenil. Revista Estudios Pedagógicos, Volumen 38, $\mathrm{N}^{\circ}$ 2. Ediciones UACh. 


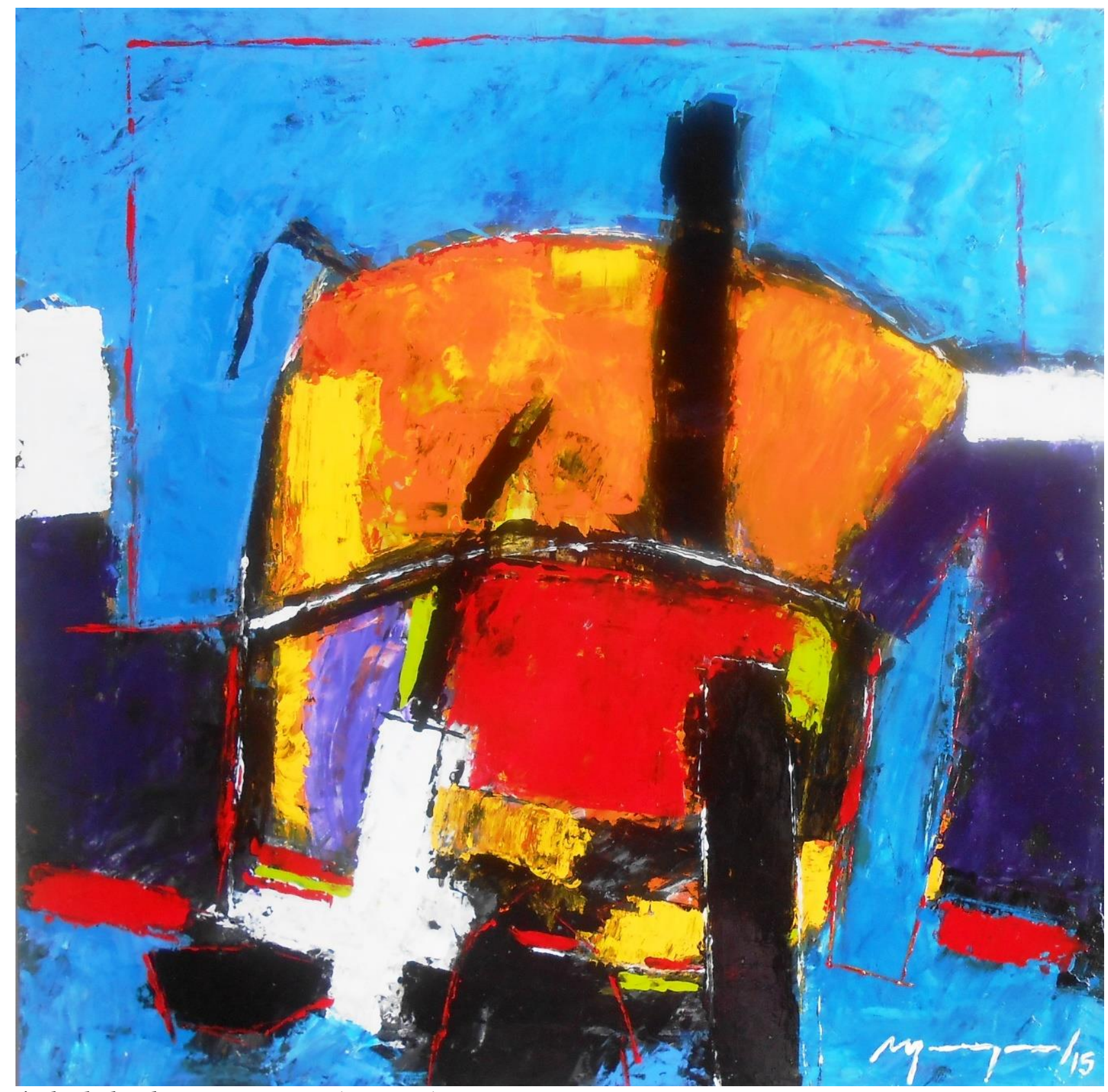

Título de la obra: Espacio vital-arqueología y paisaje

Técnica: pigmentos industriales sobre polietileno

Dimensiones de la obra: $100 \mathrm{cms} \times 100 \mathrm{cms}$

Añ $0: 2014$

Nombre de la imagen adjunta: 004 